\title{
El conocimiento profesional del profesor de biología sobre la enseñanza de la evolución
}

\author{
Roger Steve Guerrero Junca ${ }_{\text {Universidad Distrital Francisco osé de Caldas, Colombia }}^{\circledR}$
}

Autor de correspondencia: ${ }^{1}$ rsguerreroj@correo.udistrital.edu.co Recibido: 30 de agosto de 2020 Revisado: 15 septiembre de 2020 Aprobado: 31 de diciembre de 2020 Publicado: 06 de junio de 2021

\section{Resumen}

La investigación se desarrolla a partir de la propuesta de Fonseca y Martínez (2020), en la que se presenta la construcción del conocimiento profesional del profesor de biología como la integración/transformación de cinco conocimientos: disciplinar, didáctico, de la historia de vida, de la experiencia y del contexto. La metodología se basa en el análisis e interpretación en el nivel declarativo de un estudio de caso de una docente en ejercicio. Como resultado de la investigación, se evidencia que, producto de la integración/transformación de los conocimientos en la docente, emergen tres nuevos y singulares conocimientos: la enseñanza de la evolución como un vehículo en la apropiación de una actitud responsable del ser humano sobre la conservación de las especies; la enseñanza y la comprensión de la evolución biológica como mecanismo de mitigación de actitudes asociadas al machismo, la xenofobia y la discriminación sexual; y la enseñanza de la evolución y la toma de decisiones en la construcción de una ciudadanía crítica.

Palabras clave: conocimiento profesional, evolución biológica, estudio de caso 


\title{
The biology teacher's professional knowledge on the teaching of evolution
}

\begin{abstract}
The research is based on the proposal of Fonseca and Martínez (2020), in which the construction of the biology teacher's professional knowledge is presented as the integration/ transformation of five types of knowledge: disciplinary, didactic, of life history, of experience, and of context. The methodology is based on the analysis and interpretation of a case study of a practicing teacher at a declarative level. As a result of the research, it is evident that, since the teacher integrated and transformed her knowledge, three new and unique types of knowledge emerged: teaching evolution as a vehicle in the appropriation of a responsible human attitude towards the conservation of species; teaching and understanding biological evolution as a mechanism for mitigating attitudes associated with male chauvinism, xenophobia, and sexual discrimination; and teaching evolution and decision-making in the construction of critical citizenship.
\end{abstract}

Keywords: professional knowledge, biological evolution, case study

\section{O conhecimento profissional do professor de biologia sobre o ensino da evolução}

\section{Resumo}

A pesquisa é baseada na proposta de Fonseca e Martínez (2020), na qual a construção do conhecimento profissional do professor de biologia é apresentada como a integração/ transformação de cinco tipos de conhecimento: disciplinar, didático, da história da vida, da experiência e do contexto. A metodologia é baseada na análise e na interpretação a nível declarativo de um estudo de caso de uma professora em exercício. Como resultado da pesquisa, é evidente que, desde que a professora integrou e transformou seu conhecimento, três novos e únicos conhecimentos emergem: o ensino da evolução como veículo na apropriação de uma atitude humana responsável em relação à conservação das espécies; o ensino e a compreensão da evolução biológica como mecanismo para mitigar atitudes associadas ao machismo, à xenofobia e à discriminação sexual; e o ensino da evolução e a tomada de decisões na construção de uma cidadania crítica.

Palavras-chave: conhecimento profissional, evolução biológica, estudo de caso 
La investigación se enmarca en el campo de la didáctica de las ciencias. Adúriz e Izquierdo (2002) plantean que esta es una disciplina, por el momento, autónoma, centrada en los contenidos de las ciencias desde el punto de vista de su enseñanza y aprendizaje. Es una disciplina de basamento mayoritariamente epistemológico y nutrida por los hallazgos de otras disciplinas ocupadas de la cognición y del aprendizaje. Porlán et al. (1998) señalan que "la didáctica de las ciencias es una disciplina emergente posible y práctica” (p. 176). Así, el futuro de la didáctica de las ciencias ha de organizarse en torno a tres problemas: (a) profundizar en los fines y fundamentos de un modelo alternativo de enseñanza-aprendizaje de las ciencias; (b) desarrollar una nueva teoría del conocimiento escolar y de las estrategias que favorezcan su construcción; (c) desarrollar una nueva teoría del conocimiento profesional y de las estrategias que favorezcan su construcción; entre otras.

Respecto al conocimiento profesional del profesor, Porlán (2018) plantea:

Los profesores piensan y sienten en relación con la enseñanza y el aprendizaje de las ciencias y, lo que piensan y sienten es importante desde un punto de vista didáctico y formativo, es decir, también son sujetos epistémicos, portadores y constructores de significados que condicionan cualquier proceso de cambio. (p. 7)

Es decir, poseen un conocimiento profesional derivado de la integración de diversos tipos de conocimientos de orden académico y experiencial, lo que posibilita reconocer que el conocimiento profesional del profesor [CPP] se caracteriza por ser un conocimiento epistemológicamente diferenciado que deviene de la reflexión sobre la práctica.

Así, esta investigación está enmarcada en el conocimiento profesional del profesor de biología [CPPB] en la línea de la enseñanza de la evolución biológica. En las últimas décadas, una buena parte de la investigación a nivel mundial en educación ha centrado sus esfuerzos en la consolidación del conocimiento particular que tienen los profesores y que los distingue de las demás profesiones. En estas particularidades del CPP, varios autores han caracterizado sus elementos propios, como en los programas de pedagogical content knowledge -PCK- (Gess-Newsome, 1999; Grossman, 1990; Magnusson et al., 1999; Shulman, 1987, 2015), el programa del conocimiento didáctico del contenido (Parga \& Mora, 2014; Reyes \& Martínez, 2013; Valbuena, 2007), el conocimiento pedagógico del contenido (Bonilla, 2013), el CPP (Fonseca \& Martínez, 2020; Martínez el al., 2016; Pachón \& Perafán, 2013; Porlán et al., 1997), el conocimiento profesional docente (Perafán, 2015) y los saberes docentes (Tardif, 2004).

En este marco, la investigación se sustenta de acuerdo con la propuesta de Fonseca (2018), quien describe el $\mathrm{CPPB}$ como resultado dela integración/transformación decinco conocimientos: biológico, didáctico, de la experiencia, de la historia de vida y del contexto. La construcción de este conocimiento emerge de la reflexión en y sobre la práctica pedagógica, lo que permite elaborar explicaciones acerca de los fenómenos naturales de manera contextual, para aportar en la comprensión y solución de los problemas socioambientales desde una perspectiva compleja.

En cuanto a la enseñanza de la evolución, diferentes investigaciones (Araújo \& Ramírez, 2013; González, 2011; Rabino \& Audisio, 2015; Sánchez et al., 2017) evidencian la reciente apertura de esta línea de investigación y remarcan la necesidad de su abordaje. Sin embargo, las investigaciones que relacionan la enseñanza de la evolución con el CPPB son 
prácticamente inexistentes. Por tal razón, junto con el interés profesional y académico, se desarrolla esta investigación.

En el marco de la enseñanza de la biología, en las últimas décadas, han surgido distintas inquietudes respecto a la enseñanza de la evolución en el aula, pues este ha sido un tema de debate profundo a nivel académico, debido a la diversidad de implicaciones de su abordaje en lo ideológico y lo político, lo que ubica a la evolución biológica como un punto neurálgico entre el campo disciplinar y las perspectivas políticas, las representaciones del mundo propias del profesor de biología y los requerimientos institucionales. Algunos grupos de académicos latinoamericanos también han identificado la necesidad de atender dicha carencia y han avanzado en la producción de nuevo conocimiento que aborda, entre otras cosas, las dificultades y los obstáculos en la enseñanza de la evolución (González, 2011). Allí se reitera el papel fundamental y la importancia del abordaje de la evolución como eje de la enseñanza de la biología.

Por lo anterior, la investigación se planteó como objetivo caracterizar el CPPB en relación con la enseñanza de la evolución a través de un estudio de caso de una profesora en ejercicio a partir de la identificación de los cinco conocimientos descritos en la propuesta de Fonseca y Martínez (2020) y de la caracterización de su dinámica de integración/transformación. A continuación, se profundiza en los dos ejes que soportan la investigación: el CPP y la enseñanza de la evolución biológica.

\section{Respecto al conocimiento profesional del profesor}

En el marco internacional y nacional de investigación sobre el CPP, se evidencia un espectro amplio de posibilidades desde diferentes autores, en los que se encuentran algunas convergencias teóricas, pero también diversos distanciamientos en los lugares de enunciación que, para el caso colombiano, se hace cada vez más arduo, dadas las características complejas del contexto sociocultural. Sin embargo, las líneas de investigación que se han instaurado a nivel nacional evidencian un objetivo claro: el reconocimiento de un conocimiento profesional que produce el maestro al convertirse en profesional, que trasciende el lugar del cómo de la enseñanza, para aportar a la idea de un conocimiento que se produce y que se valida en la escuela (Fonseca \& Martínez, 2020).

Teniendo en cuenta el contexto colombiano en este campo, la presente investigación asume los principios del programa del CPP propuestos por Fonseca y Martínez (2020), en el que primordialmente se evidencia que en el CPP, en relación con la enseñanza, se articulan tres fuentes de conocimientos: la fuente académica - entendida desde los conocimientos particulares de la biología como disciplina, la didáctica de las ciencias, la didáctica de la biología, la naturaleza de la ciencia, el constructivismo, el pensamiento complejo y la teoría crítica-, la experiencia - manifestada como el ejercicio reflexivo que el profesor hace sobre su propia práctica y que legitima cuando le funciona de manera razonable- y el contexto - expresado en la capacidad de comprender la diversidad de estudiantes e instituciones de características particulares asociadas a la región donde estén ubicadas-. Según lo anterior, se asume que el CPP es el producto de la integración/transformación entre los siguientes conocimientos: académicos - conocimiento disciplinar y conocimiento didáctico- $y$ experienciales - historia de vida, contexto y experiencia-. 
Es necesario resaltar que el CPP es un conocimiento singular, derivado de la propiedad de integración/transformación y que su construcción depende de la intención del profesor de aportar en la formación de otros sujetos. Dada la naturaleza compleja de este conocimiento, es imperante reconocer que dicho CPP emerge de la práctica pedagógica, en la cual se produce un conocimiento de características locales e intersubjetivas, que se valida y se legitima a través de las propias transformaciones de los sujetos - maestros y estudiantesen el contexto que habitan y que posteriormente transforman en pro de una vida más digna. Así, a la enseñanza de un contenido particular le corresponde aportar en la comprensión del fenómeno de lo vivo y de la vida y, en consecuencia, del cuidado de las especies, por lo cual se promueve el cuidado de sí y del otro. En este sentido, es un conocimiento que articula desde una dimensión política, que plantea la necesidad de un conocimiento que aporte en los procesos de transformación social y del cuidado de la vida en todas sus manifestaciones.

\section{Respecto de la enseñanza de la evolución biológica}

El conocimiento científico sobre la evolución es una construcción compleja y un foco de discusión académica que se mantiene en la actualidad, dadas sus implicaciones en la comprensión de la biología en sí misma. En este sentido, el profesor de biología encuentra un primer reto académico y profesional al tener que comprender este constructo teórico, discriminar o seleccionar qué de eso entrará en su discurso en el aula, cómo lo enseñaráy cómo lo evaluará, sin contar con que esta teoría puede o no estar en concordancia con su conjunto de creencias. Lo anterior supone entonces un proceso de pensamiento, planificación, acción y reflexión, en el que no solo entra en juego la dimensión del conocimiento científico, sino, además, las dinámicas de algunos fenómenos sociales, culturales y políticos.

Como se ha mencionado antes, la evolución biológica se ha consolidado dentro de un ambiente de tensiones culturales y políticas de las que el profesor de biología no es ajeno, principalmente porque se reconoce al profesor como un sujeto inmerso en las condiciones históricas y temporales que forjarán, de una u otra manera, su sistema de creencias. Es decir, los profesores, como cualquier otro sujeto, están condicionados por su contexto cultural, que les compele a actuar y pensar de cierta manera, y a adoptar determinado sistema de valores (Mansour, 2013). En otras palabras, la forma como los individuos -en este caso los profesores de biología - piensan y actúan está moldeada por la cultura, la historia y la política. De tal forma, este sistema de creencias, conocimientos, ideas y valores configura un marco de interpretación en el cual se articula la ciencia y su enseñanza (Taber, 2013). Esto indica que la práctica de los profesores — cualquiera que esta sea- no puede comprenderse al margen de la cultura y sin referencia al contexto en el que tiene lugar (Peñaloza, 2017).

Si bien ya se mencionó que en Colombia la investigación en la enseñanza de la evolución recién ha despegado, en otras regiones del mundo se reportan avances en esta línea, de manera que ya se han descrito algunos obstáculos de los estudiantes y profesores para comprender la evolución. Varios de ellos son recogidos de la literatura y descritos por el profesor argentino González (2011) en su tesis doctoral. Aunquela mayoría de dichos obstáculos son comprendidos desde el análisis de los estudiantes, llama la atención que, en ocasiones, son transmitidos por los mismos profesores o por el contexto del educando, y no necesariamente son una creación de algún proceso realizado por el alumno (Araújo \& Ramírez, 2013).

Entrelos obstáculos señalados resaltan la presencia e influencia de valores e ideas religiosas 
tanto en estudiantes como en profesores (Griffit \& Brem, 2004; Smit, 2010, como se citó en González, 2011), el escaso conocimiento y/o la no aceptación de la teoría de la evolución por parte de los profesores de ciencias (Berkman et al., 2008; Smit, 2010, como se citó en González, 2011) y la inadecuación de los materiales y estrategias didácticos (Demastes et al., 1995; Martins \& Braga, 2002; Nehm \& Schonfeld, 2007, como se citó en González, 2011). Se pone de manifiesto que, en las decisiones que toma el profesor al enseñar la evolución, se adquieren en cierta forma - consciente o no-compromisos epistemológicos, ontológicos y axiológicos. Estos presupuestos implican, en algunas circunstancias, que conceptos o teorías como la selección natural o la evolución no se acepten, o se acojan diferencialmente (Peñaloza, 2017).

Por lo anterior, la enseñanza de la evolución biológica ha sido polémica. Los jóvenes temen desvirtuar sus creencias, ya que ha permitido no solo explicar el origen de los sistemas vivos, sino el significado de su existencia (Buskes, 2009), lo cual trasciende el ámbito científico para adentrarse en lo filosófico. Esto genera un constante debate no solo ideológico, sino también político.

\section{Metodología}

La investigación se realizó bajo los principios del enfoque cualitativo y dentro del paradigma interpretativo, el cual alude a las posturas sobre la idea de sujeto de investigación, idea de realidad y el lugar de construcción del conocimiento (Denzin \& Lincoln, 2005; Flick, 2011; Vasilachis, 2006). A su vez, se utilizó la estrategia metodológica de estudio de caso (Stake, 2008; Yin, 1994), con la cual se logra realizar un análisis riguroso de un fenómeno social particular. Para esta investigación, el fenómeno es de tipo educativo, con el que se pueden cumplir diferentes objetivos que den cuenta de nuevos hallazgos o mejoras a los marcos teóricos existentes en la investigación educativa (Páramo, 2013).

Para el análisis del estudio de caso, se seleccionó a la profesora Natalia, una docente en ejercicio que cumplía con algunas características particulares como: ser licenciada en biología, tener varios años de experiencia docente, que en el momento estuviera enseñando el componente evolutivo en sus clases y que estuviera abierta a realizar una reflexión profunda sobre su práctica. Ella autorizó de forma escrita y oral el uso de su nombre durante este ejercicio académico.

La investigación estuvo orientada al conocimiento profesional de la profesora en el nivel declarativo, es decir, lo que declara y expresa la profesora de forma oral y escrita, que se explicita a través de instrumentos como la entrevista o el diseño de unidades didácticas, previas al desarrollo de las clases (Martínez et al., 2016). Se resuelve entonces, en concordancia con los planteamientos del estudio de caso, en términos de especificidad y profundidad, desarrollar la obtención y el análisis de los datos en el nivel declarativo, mediante el análisis riguroso de la historia de vida de la profesora — narrada y recogida en una entrevista semiestructurada-. Para atender a la necesidad de complementar o triangular la información obtenida de la narrativa, se procedió a realizar un análisis de un momento específico de la práctica de la profesora: la planeación de clase.

La planeación de clase se describe como un proceso - acción de planificar- y también como producto — resultado de la planificación: guía didáctica o instrumento similar-. 
La planificación se trata entonces tanto de un proceso psicológico como de una actividad práctica (Clarck \& Peterson, 1990). Esta perspectiva de la planeación de clase trasciende entonces el nivel declarativo del profesor y se ubica en los niveles de acción y reflexión, dado que se constituye como una elaboración constante del profesor ubicada de forma atemporal en la práctica, resultado de la retroalimentación de su propia experiencia.

Por lo anterior, se establecieron cuatro fases de investigación:

1. Comprensión teórica acerca de las categorías del CPP, en relación con la enseñanza de la evolución biológica.

2. Diseño y validación de instrumentos, en la que se diseñó un instrumento de investigación —adaptado de Urazán (2017) —, basado en la entrevista semiestructurada, con el propósito de obtener información narrativa de la historia de vida de los casos de la profesora Natalia en relación con la enseñanza de la evolución biológica; además de un instrumento de revisión documental dirigido a la planeación de clase.

3. Implementación de instrumentos, en la que ejecutaron los instrumentos con la profesora Natalia y se realizó la grabación en audio de la entrevista semiestructurada y el análisis de la planeación de clase escrita. En este proceso se pusieron de manifiesto los consentimientos informados debidamente firmados y grabados en audio como lo establece la Ley 1581 de 2012 sobre la protección de datos.

4. Análisis de la información, en el que se sometió la información a un tratamiento de organización mediante el software $\mathrm{Nvivo}^{\circledR}$ para establecer categorías y rastrear los elementos presentes en el CPP de cada caso, para luego analizar detalladamente los procesos de integración/transformación.

Se comprende el CPPB como un conocimiento complejo que se construye en la integración de conocimientos académicos y experienciales, los cuales sufren procesos de transformación como una propiedad emergente de un sistema. Esto produce un conocimiento particular - el CPP sobre la enseñanza de la evolución- para este caso.

\section{Resultados y discusión}

A partir del uso del software $\mathrm{Nvivo}^{\circledR}$, se organizó y se clasificó la información recogida (tabla 1) en cinco categorías macro, correspondientes a los cinco conocimientos del CPP, y en alrededor de 39 subcategorías relacionadas directa o indirectamente con dichos conocimientos.

Los conocimientos con el mayor número de referencias y porcentaje de participación fueron: conocimiento didáctico -73: 25,6\%-, conocimiento de experiencia -69: 24,2 \%- y conocimiento biológico sobre evolución -67: 23,3\%-. Con menor número de referencias aparecen: conocimiento de contexto $-44: 15,4 \%-$ y conocimiento de historia de vida - 34: 11,9 \%-. Este resultado es considerado un indicio en la caracterización del CPP. En el caso de Natalia, se evidencia cierto grado de equilibrio en la cantidad de registros entre tres de estos conocimientos, como lo muestra la figura 1 , lo cual se logra evidenciar en el discurso sobre su práctica. 
Tabla 1

Principales subcategorías de los conocimientos del CPPB

\begin{tabular}{|c|c|c|c|c|c|}
\hline Conocimiento & Biológico & Didáctico & Del contexto & Experimental & De la historia de vida \\
\hline \multirow{4}{*}{ Subcategorías } & \multirow{2}{*}{$\begin{array}{c}\text { Familia de } \\
\text { modelos }\end{array}$} & Currículo & Aula & Contexto & Autodeterminación \\
\hline & & $\begin{array}{l}\text { Estrategias de } \\
\text { enseñanza }\end{array}$ & Institucional & Personal & Compromiso \\
\hline & \multirow{2}{*}{$\begin{array}{l}\text { Históri- } \\
\text { co-epistemo- } \\
\text { lógico }\end{array}$} & Evaluación & Nacional & Colectiva & Condiciones favorables \\
\hline & & $\begin{array}{l}\text { Finalidades de } \\
\text { la enseñanza }\end{array}$ & Sociocultural & Aula & Condiciones desfavorables \\
\hline
\end{tabular}

\section{Figura 1}

Distribución por número de referencias sobre los conocimientos de la profesora Natalia

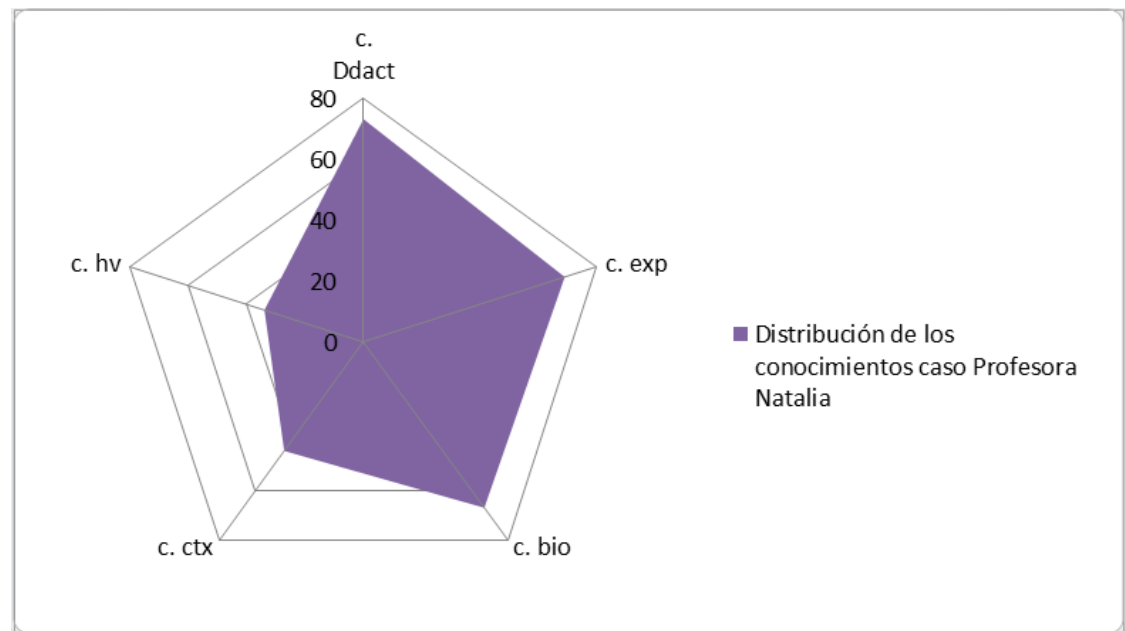

Dicho equilibrio en la cantidad de registros por conocimiento, en lo que declara la profesora Natalia y su planeación, muestra una coherencia entre la labor profesional, el conocimiento disciplinar y el conocimiento que ha construido a partir del ejercicio y de la reflexión sobre su propia práctica, de manera que se evidencia una coexistencia entre el conocimiento sobre la evolución biológica, el conocimiento didáctico y el conocimiento derivado de su experiencia como docente.

Una visualización de la distribución de las categorías internas de cada conocimiento revela la forma en la que se relacionan entre sí y consolidan una estructura general para la caracterización del CPPB sobre evolución de la profesora Natalia. En la figura 2 se evidencia la distribución de los elementos internos de cada conocimiento o subcategorías. Se destacan las finalidades de la enseñanza actitudinales y políticas para el conocimiento didáctico, las dificultades y habilidades de enseñanza en el conocimiento de experiencia, y la categoría de la evolución como mecanismo de explicación en el conocimiento biológico.

Esta distribución de subcategorías o elementos internos de los conocimientos, que hacen parte del $\mathrm{CPPB}$, se configurarán como el segundo hallazgo protagonista en la intención de caracterizar el CPPB sobre evolución.

Por otro lado, también es posible visualizar una nube de palabras que permite comprender la construcción del CPPB sobre evolución en la profesora Natalia. Entre las cincuenta palabras más frecuentes aparecen algunas previstas como evolución -100_, estudiantes 
-81-, ejemplo -61-y profesor - 49-, en concordancia con la frecuencia y distribución de referencias asociadas al conocimiento didáctico, biológico y de experiencia. Sin embargo, llama la atención en este conglomerado la aparición de palabras como social(es) —27-, mujer -18-, machismo -14- y xenofobia - 10-, elementos que de entrada parecieran ajenos al CPP sobre evolución biológica. Estos problematizan sobre el alcance y el rastreo de elementos y relaciones internas en la profesora Natalia y se consolidan como un tercer hallazgo.

\section{Figura 2}

Esquema de comparación de nodos por cantidad de referencias, producido por el software Nvivo 12

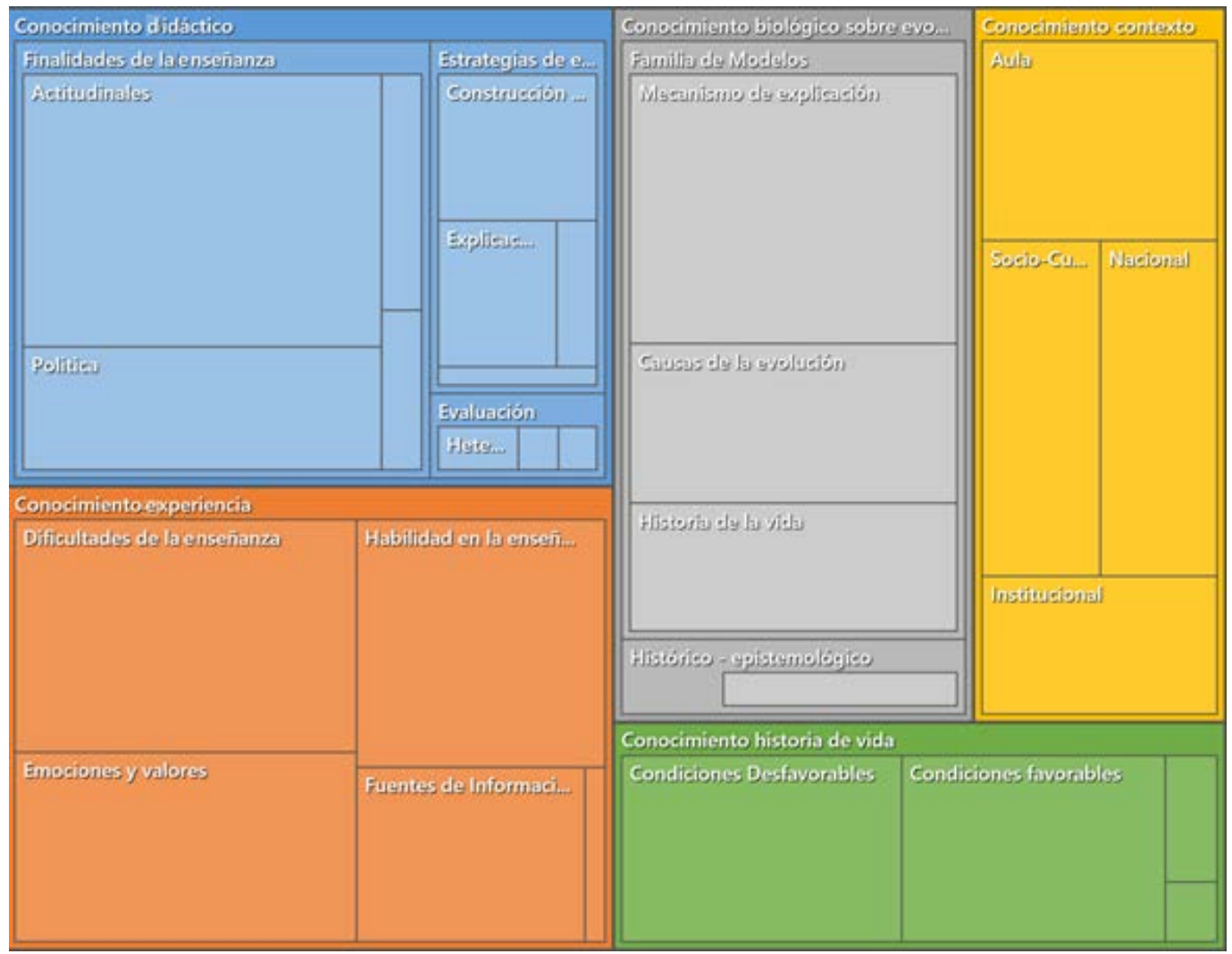

\section{Figura 3}

Nube de palabras por frecuencia en las referencias codificadas de todos los conocimientos, generada por el software Nvivo 12

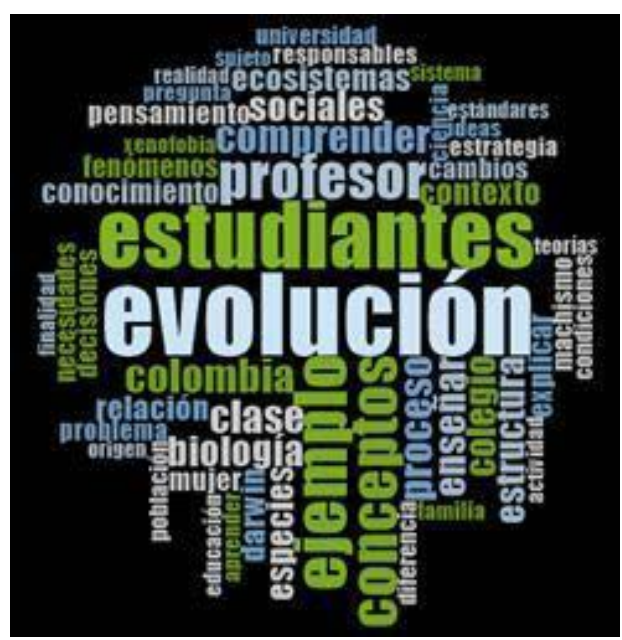


Los resultados anteriores podrían describir una aparente jerarquía en la expresión de los elementos de cada conocimiento perteneciente al CPP de la profesora Natalia. Sin embargo, Martínez et al. (2016) manifiestan sobre este tipo de resultado que la construcción del CPP no es lineal y que, por el contrario, este conocimiento es producto de tensiones de distinto orden, que contribuyen en el propio desarrollo profesional, lo cual imprime una característica de alta complejidad a la construcción y caracterización del CPP. Ejemplo de lo anterior es que para el caso de la profesora Natalia, el conocimiento sobre la historia de vida, aunque no presenta el mayor número de registros, se constituye como un conocimiento que dinamiza y tensiona la integración/transformación de los demás conocimientos en diferentes espacios temporales, y repercute en la toma de decisiones durante sus planeaciones y ejecuciones de clase.

Se resalta entonces la cantidad de elementos no solo al interior del CPPB, sino del contexto de lugar, tiempo y espacio en donde se construye. En un primer ejercicio de comprensión de la complejidad del CPPB, posterior a la identificación de sus conocimientos, se caracterizaron las relaciones que se evidencian entre los conocimientos, que corresponden a las categorías y subcategorías de análisis previas, y cuyo análisis es el conocimiento base en la construcción de categorías emergentes propias de la caracterización del CPPB sobre evolución.

Un análisis exhaustivo de la forma en que emergen las relaciones entre los conocimientos da cuenta del proceso de integración/transformación de los conocimientos que constituyen el CPP sobre evolución en el caso de la profesora Natalia, además de la forma en que se movilizan internamente dichos conocimientos. En concordancia con los hallazgos anteriores, resalta la abundancia de relaciones entre los conocimientos didáctico, de experiencia y biológico, por medio de elementos como las estrategias y finalidades de la enseñanza, actitudinales y políticas, las fuentes de información y la evolución como mecanismo de explicación, respectivamente.

Un ejemplo de esta integración de conocimientos se evidencia en la relación entre la historia de vida y el conocimiento biológico sobre evolución, en donde destaca la formación académica y familiar de corte laico desde la primera infancia de la profesora Natalia, elemento que se va a reflejar posteriormente en su construcción de realidad y en su comprensión de la evolución biológica. Esto se evidencia en su narrativa de historia de vida.

Sobre su construcción de realidad en relación con la formación laica y la comprensión de la evolución biológica, la profesora Natalia recuerda:

- Cómo te decía, yo vengo de una educación laica. Mi comprensión del mundo ha estado atravesada por muchas cosas, pero es hasta que entiendo la biología que encuentro un sentido para comprenderlo, y pues parte de esa comprensión es gracias al concepto de evolución. De cierta forma la evolución ha sido un aporte muy grande en la forma en la que yo comprendo la realidad, nunca he chocado con eso, además porque es entendible, demostrable y le aporta mucho a la explicación que tengo del mundo.

Respecto a lo anterior, Puyana y Barreto (1994) resaltan que la reconstrucción de la historia de vida ofrece amplias posibilidades para el conocimiento y análisis de los complejos procesos de construcción de identidad. También mencionan que la narración y la reflexión sobre las vivencias hacen posible el encuentro entre el tiempo del sujeto y el tiempo del 
mundo, entre la finitud de la vida personal y la infinitud de la historia social humana.

Dada la integración/transformación de los conocimientos de la profesora Natalia, se caracterizan tres conocimientos emergentes, como lo describe la figura 4.

\section{Figura 4}

Conocimientos emergentes a partir de la integración/transformación de los cinco conocimientos

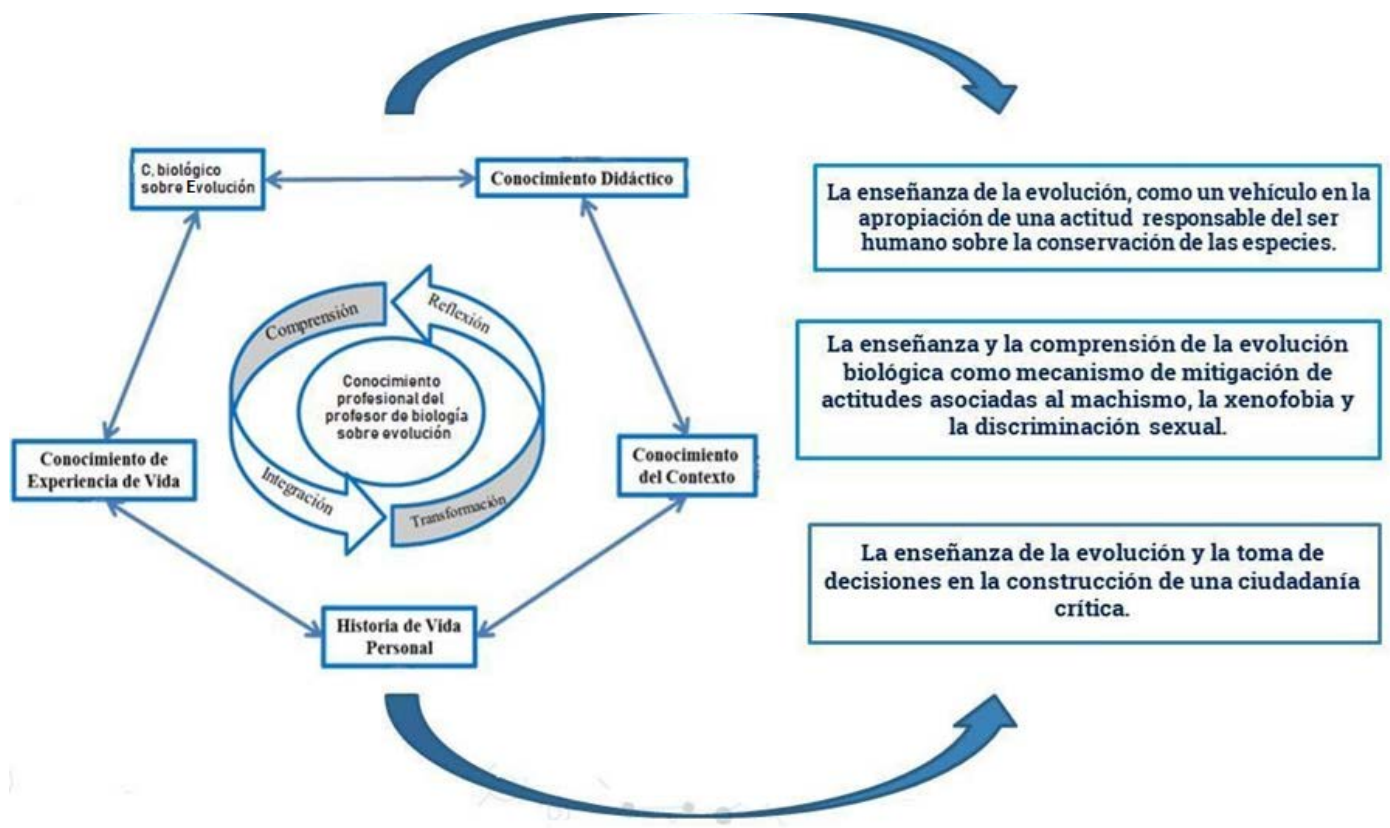

Debido a la extensión de los resultados de esta integración/transformación, se presentará a profundidad uno de los tres conocimientos emergentes: la enseñanza y la comprensión de la evolución biológica como mecanismo de mitigación de actitudes asociadas al machismo, la xenofobia y la discriminación sexual. Los otros conocimientos emergentes serán descritos en futuras publicaciones.

\section{La enseñanza y la comprensión de la evolución biológica como mecanismo de mitigación de actitudes asociadas al machismo, la xenofobia y la discriminación sexual}

A continuación se describen, la integración de los diversos conocimientos que hacen posible la emergencia del pensar la enseñanza de la evolución biológica que promueve una reflexión en relación con las actitudes de machismo, xenofobia y discriminación sexual.

\section{Integración del conocimiento biológico en clave del conocimiento didáctico y experiencial}

Respecto a la relación entre las finalidades de la enseñanza de tipo actitudinal y comprensión de la evolución biológica y el machismo, en la entrevista sobre el conocimiento biológico, la profesora Natalia expresa:

- Entonces también hay que tener cuidado cómo entendemos y enseñamos evolución. Me parece que viéndonos como especie y viéndonos como organismos igual de importantes en la especie, hombre y mujer, donde ambos somos productos de un proceso evolutivo, entonces de ahí se pueden sacar muchas cosas para que los estudiantes 
reflexionen, porque ahora se están viviendo los extremos violentos del machismo, pero también de una corriente donde algunas mujeres creen que pueden ser superiores a los hombres y responden con violencia [...] las funciones, por ejemplo, que un niño comprenda el ciclo hormonal de las mujeres y la menstruación y con eso baje ese machismo horrible que tenemos en Colombia me parece hermoso.

- De pronto encuentro más evidente la relación evolución-racismo y evoluciónmachismo. Entonces, ahí hay un reto de pensarse todos los días en todas las clases. ¿Cómo hago para que estos fenómenos se detengan o se vayan diluyendo?

- Y es interesante cómo después de esas clases se generan unos cambios, y se explican cosas sobre menstruación. Luego los niños hombres son los que explican y se termina generando una eliminación de micromachismos como palabras de "veintiochuda", y dicen: "No pues es que yo entiendo lo que está pasando. Se está rompiendo su útero y está botando sangre arterial. Claro, yo también estaría de mal genio".

La presente investigación asume como machismo a la exteriorización y significación, fruto de la ideología patriarcal. El machismo es conformado por todas las acciones y omisiones, visibles o invisibles, que sustentan la opresión de las mujeres (Herreros, 2017) en el marco de la obsesión masculina con el predominio y la virilidad, que tiene expresión en la posesividad respecto de la propia mujer y en actos de agresión y jactancia en relación con otros hombres (Viveros-Vigoya, 2006).

Respecto a este hallazgo, existe una discusión académica. El historiador israelí Yuval Noah (2014, como se citó en Merchán, 2016), en una de sus teorías acerca del origen biológico del machismo, menciona que:

La evolución ha programado a los varones para el machismo, porque según la teoría de la selección natural de Darwin, los hombres entraron en una competencia por tener descendencia, lo que de manera natural asignó a las mujeres el rol de sumisas y cuidadoras. (p. 39)

El anterior planteamiento revela de lleno una aparente justificación evolutiva del machismo. Sin embargo, ante esa aseveración Bertranpetit (como se citó en Querol, 2015) responde:

No hay duda de las diferencias entre sexos, sean físicas o de comportamiento; en humanos estas diferencias son pequeñas pero evidentes. Pero eso no implica que estas diferencias lleven a situaciones de poder social. La biología nos muestra cómo son las cosas. Y es importante saberlas porque entonces culturalmente podemos cambiar nuestro comportamiento para adecuarlo a nuestros propósitos. (párr.11)

Esta última frase puede interpretarse como un inminente llamado al profesorado de biología en dos aspectos: a la correcta comprensión e interpretación del conocimiento biológico sobre evolución; y a su enseñanza intencionada, es decir, a hacer uso de la biología evolutiva para mitigar el machismo y no para justificarlo.

Mayr (2006), en su texto ¿Por qué es única la biología?, expone que la evolución es un fenómeno que, por su complejidad, puede ser abordado para el análisis y la comprensión de fenómenos sociales y, en general, para la comprensión del ser humano. Este hecho se 
evidencia una y otra vez en la comprensión del conocimiento biológico sobre evolución y toma ahora mayor relevancia en el marco de su enseñanza, situación evidenciada en gran manera por la profesora Natalia en las unidades de registro. Allí hace especial énfasis en las relaciones entre las explicaciones evolutivas y las actitudes de los estudiantes cuando se enfrentan a situaciones cotidianas relacionadas con las violencias basadas en género, todo ello dentro del contexto cultural colombiano, en el que se percibe el machismo como un elemento transgresor de la paz, como se constata en el informe de violencia contra las mujeres de la ONU Mujeres (2016), basado en datos del consolidado de Medicina Legal, en el que se establece que el $50 \%$ de los casos de violencia de pareja contra las mujeres en Colombia se debe a intolerancia y machismo.

\section{Integración del conocimiento de contexto en clave del conocimiento biológico y didáctico}

Respecto al racismo y la xenofobia en relación con la enseñanza de la evolución, la profesora Natalia menciona:

- En el caso del racismo y la xenofobia la evolución es perfecta [...] Entonces también entra que desde de la evolución uno puede explicar que un ser humano no se debe sentir superior a otro, porque no tiene sentido. Entonces, ahí el tema del racismo y la xenofobia se caen.

- Claro que sí puede aportar a todos. Estos fenómenos ya están presentes. Entonces, lo primero que hay que tratar de averiguar es el origen para que no se sigan presentando, en el caso de la xenofobia, por ejemplo, y más en el contexto colombiano.

Al igual que el machismo, el racismo y la xenofobia son expresiones sociales que afectan la dinámica social en el contexto colombiano. Las principales víctimas de la discriminación racial son los miembros de los pueblos indígenas y las comunidades afrodescendientes (ONU, 2009). La comprensión del momento histórico y contextual por parte de la profesora Natalia moviliza en gran modo las decisiones que toma en el aula respecto a la finalidad de la enseñanza de la evolución biológica. Ratifica la necesidad de enseñar biología como manera de aportar en la mitigación de fenómenos sociales, en este caso el racismo y la xenofobia.

Como ya se dijo, una particularidad de estas expresiones sociales negativas es que han encontrado de algún modo una aparente justificación en el ámbito biológico. Históricamente se ha relacionado la teoría evolutiva con las corrientes supremacistas raciales. Sánchez (2007) propone la existencia de una conexión ideológica entre las teorías evolutivas sobre las llamadas "razas humanas" - especialmente los modelos darwinistas sobre evolución humana - y las jerarquías raciales, lo cual establecía la lógica económica del imperialismo occidental. Sobre esta línea, otros autores han expuesto:

La idea moderna de la raza aparece a partir del siglo XVIII con el denominado racismo científico que planteaba la existencia de diferencias esenciales y naturales entre los grupos humanos y que, de esta forma, generó jerarquías sociales a partir de particularidades fenotípicas consideradas como diferencias biológicamente esenciales. La producción de estas teorías estuvo acompañada de explicaciones científicas sobre la inferioridad de unas razas frente a otras y la legitimidad de la dominación de aquellas que se suponían inferiores por parte de las superiores. (Agudelo, 2002, como se citó en Quintero, 2013, p. 74) 
Sin embargo, en la actualidad pareciera que hay un común acuerdo en la no aceptación de la raza como una categoría científica fundamentada en cualquier diferencia biológica, sino como una construcción social, histórica e ideológicamente definida (Quintero, 2013). De tal forma, el racismo nos remite a configuraciones específicas de dominación social, fundamentadas en la idea de la raza, de acuerdo con determinados contextos sociales, históricos, ideológicos, culturales y geográficos. Dicho de otra forma, los racismos deben ser ubicados en sus contextos espaciales y temporales (Wade, 1997, como se citó en Quintero, 2013).

La idea anterior nos remite entonces a la necesidad imperante de acciones que permitan esclarecer la inexistencia de una base biológica y evolutiva del racismo. Se debe reivindicar que la comprensión de la biología evolutiva permitiría reducir la base ideológica en la que se sustenta, momento en el cual los profesores de biología debemos acudir como actores y sujetos de transformación social, en el marco de un análisis crítico de nuestra realidad nacional.

Por último, en referencia a la relación de las finalidades de la enseñanza de la evolución y el conocimiento biológico, la profesora Natalia reconoce la discriminación sexual como un elemento susceptible de análisis desde la clase de evolución y expresa:

- Incluso, en ese grupo de fenómenos sociales que se podrían atender, también ingresa el de diversidad sexual. En el contexto colombiano, ha resultado bien problemático por la discriminación [...] Ahora el problema es más complejo, porque entonces estas personas con estas creencias se reúnen y ubican a un líder qué va a tomar decisiones políticas, y una cantidad de expresiones violentas, como discriminar a los homosexuales [...] Entonces, claro, ahí entra la enseñanza de la evolución, entra a participar y a ayudar, entra a ser base junto con la genética, por ejemplo. Son conceptos que entran a apoyar y a decir —carambas- que somos distintos incluso genéticamente.

Sobre la diversidad sexual, dada su complejidad, existen diferentes líneas de investigación y desde diversas áreas del conocimiento. La biología evolutiva en particular ha hecho presencia en el intento de darle explicación. Autores, como Soler (2005), reconocen que

el paradigma darwiniano de la genética ha extendido su influencia a las ciencias sociales y este es más claramente visto en el surgimiento de nuevas disciplinas como la psicología evolutiva, que sostiene que la conducta humana puede ser entendida en términos de su valor adaptativo; así, la conducta exhibida por los humanos ha contribuido a la supervivencia y reproducción en el pasado evolutivo y ha perpetuado los genes que influyen la conducta. Este paradigma evolutivo ha incrementado visiblemente su influencia a muchas áreas de estudio de la conducta humana, y la orientación sexual no puede ser la excepción; sin embargo, es claro que históricamente la homosexualidad no puede ser explicada bajo un solo modelo, dado la complejidad de los factores que influyen en su desarrollo. (p. 162)

Ahora, si bien la investigación sobre la diversidad sexual aún está desarrollándose, es claro que, para el contexto colombiano, los casos de agresiones violentas a personas con preferencias sexuales diversas son una realidad, y la escuela no es una excepción. En respuesta a ello, es evidente, en el desarrollo discursivo de la profesora Natalia, que el ejercicio didáctico en la enseñanza de la evolución biológica dirige su atención hacia la contención de las posibles actitudes discriminatorias por parte de los y las estudiantes, 
basadas en las preferencias sexuales de las personas; y hacia la proyección del concepto de diversidad, de forma que trascienda el campo de lo biológico y sea concebido en el campo del desarrollo personal y social.

\section{Integración del conocimiento de historia de vida en clave del conocimiento didáctico}

El conocimiento didáctico se ubica en el marco de la didáctica, como una disciplina científica (Astolfi, 2001), que problematiza los procesos de enseñanza y de aprendizaje de los conocimientos científicos derivados de las investigaciones en cada una de las áreas del conocimiento. En este sentido, A. Zambrano (2016) plantea que esta ciencia aporta en la comprensión acerca de "la génesis, circulación y apropiación del saber y sus condiciones de enseñanza y aprendizaje" (p. 57). El autor amplía que la didáctica se especifica en tres direcciones: "como disciplina científica, como sistema de aprendizaje y como espacio de investigación en las ciencias de la educación” (p. 167). En estas especificidades, L. Zambrano (2005) enfatiza que la didáctica tiene la tarea de "comprender los saberes que la ciencia produce [...] desarrollar la manera y el método para acceder de modo directo y extraordinario al patrimonio de los saberes" (p. 169). En esta misma dirección de relacionar la didáctica con el saber, Castaño y Fonseca (2008) la reconocen "como un campo de saber que se ocupa de reflexionar y teorizar sobre la práctica pedagógica en relación con los procesos de enseñanza y los procesos de aprendizaje" (pp. 84-85). De esta manera, dentro del conocimiento didáctico, la didáctica se articula con las finalidades de la enseñanza y del aprendizaje, las cuales, para el caso de la profesora Natalia, están atravesadas por las construcciones ideológicas, políticas y culturales, y, por lo tanto, de su historia de vida.

Un elemento que destaca la profesora Natalia en esta relación es la profundidad a la que llega un evento de su vida personal y que trasciende sobre su manera de pensar la educación y la enseñanza, a tal punto que se establece como un acto que impacta su concepción de finalidad de la educación. En un relato sobre su preadolescencia, la profesora Natalia recuerda:

- Entonces él me mostró que uno de profe debe defender a sus estudiantes, porque a muchos chicos no les pasa eso. Ni siquiera los defienden en la casa. Entonces, me di cuenta de que los profesores tenemos una responsabilidad muy grande, también de defender a los niños, y yo también quería hacer eso. Ahora quiero encontrar las herramientas para eso, para defender, porque infortunadamente no todas las personas que pasan por eso corren con la misma suerte, porque ni los profesores ni su familia hacen nada. Entonces, sí creo que una labor del profesor es defender, porque antes de ser profesor o estudiante somos sujetos y si no nos defendemos entre nosotros, ¿̇entonces qué?

Es importante señalar que esta defensa como finalidad de la enseñanza y del aprendizaje se articula con las propias construcciones conceptuales, procedimentales y actitudinales, en relación con la comprensión de los conceptos estructurantes de la biología. Al respecto, Natalia plantea:

- Yo creo que la biología es una ciencia que puede atravesar por todas las esferas de un sujeto. Entonces, por ejemplo, desde una dimensión organísmica, pues él puede comprender que como producto de la evolución tiene unas características y 
un funcionamiento. Entonces, con ese conocimiento, pues él también puede tomar decisiones responsables de su cuerpo, por ejemplo. Ahora, también cuando la persona se da cuenta que puede tomar decisiones que no solo tienen que ver con él, sino con los demás, la biología es súper importante para eso, por ejemplo, estar a favor o en contra de la eutanasia. Entonces, la biología entra a dar muchos argumentos que pueden terminar en una decisión o en otra, estar a favor o estar en contra y poder tomar decisiones políticas, pero, además de eso, la biología también atraviesa las esferas sociales del sujeto. Por eso considero que es tan importante enseñarla y aprenderla.

Como se puede ver, un evento personal durante su época escolar, que puede considerarse traumático, queda inserto en la mente de la profesora hasta la actualidad y emerge en su discurso para referirse a lo que debe hacer un profesor en el ámbito de las relaciones humanas con sus estudiantes, discurso que está dirigido a la defensa de sus estudiantes, ya sea por condiciones individuales de los estudiantes o, como en este caso, por la pertenencia a minorías étnico-raciales o de género. Esta situación es descrita por Caparrós y Sierra (2012), quienes mencionan que "a menudo determinadas cuestiones abiertas en nuestras biografías - en tanto que las arrastramos- están presentes en quienes somos como educadores y como investigadores" (p. 61).

\section{Conclusiones}

El CPPB sobre evolución, en el caso de la profesora Natalia, se caracteriza a partir de la integración/transformación de cinco conocimientos base descritos por el programa del profesor Fonseca (2018): el conocimiento biológico, el conocimiento didáctico, el conocimiento de su propia historia de vida, el conocimiento de la experiencia y el conocimiento del contexto. Producto de esta integración/transformación de conocimientos, junto con las diversas relaciones e interacciones, se logran describir tres conocimientos emergentes en relación con la enseñanza de la evolución: la enseñanza de la evolución, como un vehículo en la apropiación de una actitud responsable del ser humano sobre la conservación de las especies; la enseñanza y la comprensión de la evolución biológica como mecanismo de mitigación de actitudes asociadas al machismo, la xenofobia y la discriminación sexual; y la enseñanza de la evolución y la toma de decisiones en la construcción de una ciudadanía crítica.

La discusión académica sobre las bases biológicas del machismo, el racismo, la diversidad sexual y su discriminación no es un tema nuevo. Sin embargo, el abordaje de este fenómeno desde la enseñanza de la biología, y particularmente desde la enseñanza de la evolución biológica, abre una línea sobre la cual pueden y deben dirigirse las miradas en la investigación educativa, donde los profesores y las profesoras de biología debemos hacernos partícipes en la construcción y consolidación de actitudes ciudadanas responsables basadas en el respeto y el reconocimiento del otro, como otro legítimo y como sujeto de derechos, cualquiera sea su condición ideológica, política o sexual.

Se puede evidenciar que en el caso de los y las profesoras de biología, y por extensión en la enseñanza de la evolución, el docente actúa como una puerta de acceso a la información, en donde reconoce a su estudiante como sujeto que construye su conocimiento, el cual, de forma crítica, tomará decisiones sobre sí mismo y sobre los demás. 
Con lo anterior, se pone en evidencia que el profesor de biología en el ejercicio de la enseñanza de la evolución se constituye como un actor que aporta a la construcción de ciudadanos críticos y libres capaces de tomar decisiones, producto de las capacidades reflexivas y mediadas por el conocimiento construido en su relación con los otros.

\section{Referencias}

Adúriz-Bravo, A. \& Izquierdo, M. (2002). Acerca de la didáctica de las ciencias como disciplina autónoma. Revista Electrónica de Enseñanza de las Ciencias, 1(3), 130-140.

Araujo. R. y Ramírez L. (2013) Obstáculos al aprendizaje del concepto estructurante evolución biológica. Bio-grafía Escritos sobre la Biología y su Enseñanza, (edición extraordinaria), $231-244$.

Astolfi, J. (2001). Conceptos clave en la didáctica de las disciplinas. Díada Editora.

Bonilla, O. (2013). Ampliando la conceptualización del conocimiento pedagógico del contenido, la perspectiva intercultural [Tesis Doctoral, Universidad de Antioquia]. Repositorio Institucional Universidad de Antioquia. https:// bibliotecadigital.udea.edu.co/bitstream/10495/4831/1/BonillaLopezOmarAlonso_2014_ ConceptualizacionConocimientoPedagogicoIntercultural.pdf

Buskes, C. (2009). La herencia de Darwin. La evolución en nuestra visión del mundo. Herder.

Caparrós, E., \& Sierra, J. (2012). Al hilo de lo vivido: la pedagogía como brújula para la investigación. En J. Rivas, F. Hernández, J. Sancho, \& C. Núñez (Eds.), Historias de vida en educación: sujeto, diálogo, experiencia (pp. 61-66). Red Universitaria de Investigación Innovación Educativa (REUNI+D).

Castaño, C., \& Fonseca, G. (2008). La didáctica: un campo de saber y de prácticas. En C. Rozo, R. Reyes, P. Ortega, G. Fonseca, C. Castaño, R. Medina, \& D. Peñuela, Contextos y pretextos sobre pedagogía (pp. 73-95). Fondo Editorial UPN.

Clark, C., \& Peterson P. (1990). Procesos de Pensamiento de los docentes. En M. Wittrock (Ed.). La investigación de la enseñanza III. Profesores y alumnos (pp. 444-531). Paidós Educador.

Denzin, N., \& Lincoln, Y. (2005). Manual de investigación cualitativa. Vol. I. Gedisa.

Flick, U. (2011). Introducción a la investigación cualitativa. Morata.

Fonseca, G. (2018). Los ejes DOC: una estrategia conceptual y metodológica en la construcción del conocimiento profesional del profesor. Revista Científica, 31(1), 68-84. https://doi. org/10.14483/23448350.12432

Fonseca, G., \& Martínez, C. (2020). El conocimiento profesional del profesor: una construcción desde la integración/transformación de referentes académicos y experienciales. El caso de un futuro profesor de biología. En A. Molina (Ed.), Investigación y formación de profesores de ciencias: diálogos de perspectivas latinoamericanas (pp. 131159 ). Universidad Distrital Francisco José de Caldas.

Gess-Newsome, J. (1999). Secundary Teachers' Knowledge and beliefs about Subjet Matter and their Impact on Instruction. En J. Gess-Newsome, \& N. Lederman (Eds.), Examining 
Pedagogical Content Knowledge. The Construct and its Implications for Science Education (pp. 51-94). Kluwer Academic Publishers.

González, G. (2011). Obstáculos para el aprendizaje del modelo de evolución por selección natural. [Tesis Doctoral, Universidad de Buenos Aires] https://bibliotecadigital.exactas. uba.ar/collection/tesis/document/tesis_n4961_GonzalezGalli

Grossman, P. (1990). The making of a teacher: Teacher knowledge \& teacher education. Teachers College Press.

Herreros, I. (2017). Patriarcado, machismo y misoginia: reproche penal [Curso de Formación de Fiscales Mujer e Igualdad]. Centro de Estudios Jurídicos, Fiscalía Provisional de las Palmas, Islas Canarias, España. https:/elpais.com/elpais/2015/03/13/ mujeres/1426223100_142622.html

Colombia. (2012, 17 de octubre). Ley estatutaria 1581 de 2012. Diario Oficial No. 48.587. http://www.secretariasenado.gov.co/senado/basedoc/ley_1581_2012.html

Magnusson, S., Krajcik, J., \& Borko, H. (1999). Nature, sources and development of pedagogical content knowledge. En J. Gess-Newsome, \& N. Lederman (Eds.), Examining pedagogical content knowledge (pp. 95-132). Kluwer Academic Publishers.

Mansour, N. (2013). Science Teachers' cultural beliefs and diversities: A sociocultural perspective to science education. En N. Mansour, \& R. Wegerif (Eds.), Science education for diversity (pp. 205-230). Springer.

Martínez, C., Álvarez, E., \& Torres, L. (2016). El conocimiento profesional del profesor(a) de ciencias sobre el conocimiento escolar: caso de la profesora Luz en aulas vivas. En Martínez, C. (Ed.), El conocimiento profesional del profesor(a) de ciencias sobre el conocimiento escolar: Dos estudios de caso, en aulas vidas y aulas hospitalarias del distrito capital de Bogotá (pp. 61-84). Centro de Investigaciones y Desarrollo Científico; Universidad Distrital Francisco José de Caldas; Doctorado Interinstitucional en Educación.

Mayr, E. (2006). ¿Por qué es única la biología? Consideraciones sobre la autonomía de una disciplina cientifica. Kats Editores.

Merchán, A. (2016). ¿Puede rastrearse el origen del machismo? Revista Para el Aula - IDEA, $18,39-40$.

ONU Mujeres. (2016). Colombia: violencia contra las mujeres. https://umaic.org/ snapshots/170420Snapshot_mujeres.pdf

ONU. (2009). Discriminación racial en Colombia: informe alterno ante el Comité para la Eliminación de la Discriminación Racial de la ONU. Programa de Justicia Global y Derechos Humanos.

Pachón, J., \& Perafán, G. (2013). El conocimiento profesional del profesor de educación física. Lúdica pedagógica, 2(18), 23-131.

Páramo, P. (2013). La investigación en ciencias sociales: estrategias de investigación. Universidad Piloto de Colombia. 
Parga, L., \& Mora, P. (2014). El PCK, un espacio de diversidad teórica: conceptos y experiencias unificadoras en relación con la didáctica de los contenidos en química. Educación Química, 25(3), 332-342.

Peñaloza, G. (2017). Relaciones ciencia-religión y enseñanza de la evolución; estudio de casos con profesores de biología de educación básica secundaria en Colombia [Tesis Doctoral, Universidad Distrital Francisco José de Caldas y Universidad Federal de Bahía]. Repositorio Institucional Universidad Distrital - RIUD. https://repository.udistrital.edu. co/handle/11349/6413

Perafán, G. (2015). Conocimiento profesional docente y prácticas pedagógicas. Aula Humanidades.

Porlán, R. (2018). Didáctica de las ciencias con conciencia. Enseñanza de las Ciencias, $36(3), 5-22$.

Porlán, R., Rivero, A., \& Martín, R. (1998). Conocimiento profesional y epistemología de los profesores II: estudios empíricos y conclusiones. Enseñanza de las Ciencias, 16(2), 271-288.

Porlán, R., Rivero, G., \& Martín, R. (1997). Conocimiento profesional y epistemología de los profesores I. Teoría, métodos e instrumentos. Enseñanza de las Ciencias, 15(2), 155-171.

Puyana, Y., \& Barreto, J. (1994). La historia de vida: Recurso en la investigación cualitativa Reflexiones metodológicas. Maguaré, (10), 186-195. http://www.bdigital.unal.edu. co/18451/2/14265-48104-1-PB.pdf

Querol de, R. (2015). No pregunten a Darwin: tres teorías sobre el origen del machismo. Diario El País. https://elpais.com/elpais2015/03/13/mujeres/1426223100_142622.html

Quintero, O. (2013). El racismo cotidiano en la universidad colombiana desde la experiencia vivida por los estudiantes negros en Bogotá. Universitas Humanística, 77, 71-94.

Rabino, M., \& Audisio, E. (2015). Conocimiento didáctico del contenido sobre el origen de la vida e inicio de la evolución biológica en dos docentes en formación. Universidad Nacional de Mar del Plata.

Reyes, J., \& Martínez, C. (2013). Conocimiento didáctico del contenido en la enseñanza del campo eléctrico. TED, 33, 37-60.

Sánchez, J. (2007). La biología humana como ideología: el racismo biológico y las estructuras simbólicas de dominación racial a fines del siglo XIX. Theoria, 61, 107-124.

Sánchez, J. Conde, M. y Zapata, V. (2017). Concepciones alternativas sobre evolución. Un estudio en futuros maestros. Enseñanza de las Ciencias, (número extraordinario), 22192224..

Shulman, L. (1987). Knowledge and teaching: Foundations of the new reform. Harvard Educational Review, 57(1), 1-22.

Shulman, L. (2015). Shulman, L. (2015). PCK Its genesis and exodus. En A. Berry, P. Friedrichsen, \& J. Loughran (Eds.), Re-examining Pedagogical Content Knowledge in 
Science Education (pp. 3-13). Norman \& Lederman.

Soler, F. (2005). Evolución y orientación sexual. Diversitas: Perspectivas en Psicología, 1(2), 161-173.

Stake, R. (2008). Investigación con estudio de caso. Morata.

Taber, K. (2013). Conceptual frameworks, metaphysical commitments and worldviews: The challenge of reflecting the relationships between science and religion in science education. En N. Mansour, \& R. Wegerif (Eds.), Science education for diversity (pp. 151177). Springer.

Tardif, M. (2004). Los saberes del docente y su desarrollo profesional. Narcea.

Urazán, R. (2017). Conocimiento profesional específico de los profesores de biología asociado a la noción de gen [Tesis de Maestría, Universidad Pedagógica Nacional]. Repositorio Institucional UPN. http://repository.pedagogica.edu.co/handle/20.500.12209/7807

Valbuena, E. (2007). El conocimiento didáctico del contenido biológico: estudio de concepciones disciplinares y didácticas de futuros docentes de la Universidad Pedagógica Nacional [Tesis Doctoral, Universidad Complutense de Madrid].E-Prints Complutense. https://eprints. ucm.es/id/eprint/7731/

Vasilachis, I. (2006). Estrategias de investigación cualitativa. Gedisa.

Viveros-Vigoya, (2006). El machismo latinoamericano: un persistente malentendido. En M. Viveros, C. Rivera, \& M. Rodríguez (Comps.), De mujeres, hombres y otras ficciones: género y sexualidad en América Latina (pp. 111-128).Tercer Mundo Editores; Universidad Nacional, Facultad de Ciencias Humanas

Yin, R. (1994). El estudio de caso en investigación: diseño y métodos. Sage Publicaciones.

Zambrano, A. (2016). Pedagogía y didáctica: esbozo de las diferencias, tensiones y relaciones de dos campos. Praxis \& Saber, 7(13), 45-61.

Zambrano, L. (2005). Didáctica, pedagogía y saber. Magisterio. 